This item was submitted to Loughborough's Research Repository by the author.

Items in Figshare are protected by copyright, with all rights reserved, unless otherwise indicated.

\title{
Markov-chain model of classified atomistic transition states for discrete kinetic Monte Carlo simulations
}

PLEASE CITE THE PUBLISHED VERSION

http://dx.doi.org/10.1103/PhysRevE.84.046714

PUBLISHER

(C) American Physical Society

VERSION

VoR (Version of Record)

LICENCE

CC BY-NC-ND 4.0

REPOSITORY RECORD

Numazawa, Satoshi, and Roger Smith. 2019. "Markov-chain Model of Classified Atomistic Transition States for Discrete Kinetic Monte Carlo Simulations". figshare. https://hdl.handle.net/2134/15409. 
This item was submitted to Loughborough's Institutional Repository (https://dspace.lboro.ac.uk/) by the author and is made available under the following Creative Commons Licence conditions.

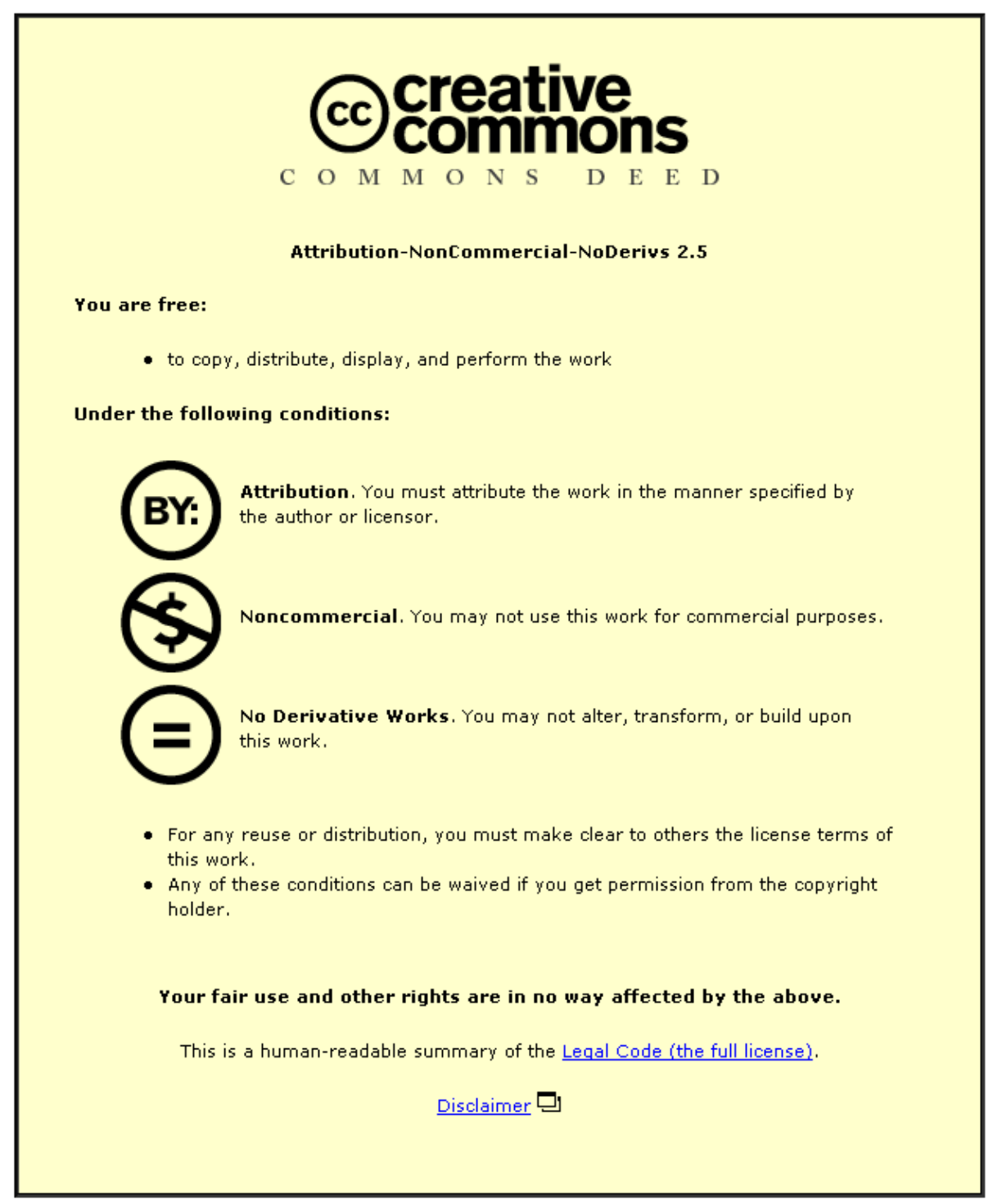

For the full text of this licence, please go to: http://creativecommons.org/licenses/by-nc-nd/2.5/ 


\title{
Markov-chain model of classified atomistic transition states for discrete kinetic Monte Carlo simulations
}

\author{
Satoshi Numazawa* and Roger Smith ${ }^{\dagger}$ \\ Helmholtz-Zentrum Dresden Rossendorf (HZDR), PO Box 510119, D-01314 Dresden, Germany \\ (Received 3 February 2011; revised manuscript received 9 August 2011; published 31 October 2011)
}

\begin{abstract}
Classical harmonic transition state theory is considered and applied in discrete lattice cells with hierarchical transition levels. The scheme is then used to determine transitions that can be applied in a lattice-based kinetic Monte Carlo (KMC) atomistic simulation model. The model results in an effective reduction of KMC simulation steps by utilizing a classification scheme of transition levels for thermally activated atomistic diffusion processes. Thermally activated atomistic movements are considered as local transition events constrained in potential energy wells over certain local time periods. These processes are represented by Markov chains of multidimensional Boolean valued functions in three-dimensional lattice space. The events inhibited by the barriers under a certain level are regarded as thermal fluctuations of the canonical ensemble and accepted freely. Consequently, the fluctuating system evolution process is implemented as a Markov chain of equivalence class objects. It is shown that the process can be characterized by the acceptance of metastable local transitions. The method is applied to a problem of $\mathrm{Au}$ and $\mathrm{Ag}$ cluster growth on a rippled surface. The simulation predicts the existence of a morphology-dependent transition time limit from a local metastable to stable state for subsequent cluster growth by accretion. Excellent agreement with observed experimental results is obtained.
\end{abstract}

DOI: 10.1103/PhysRevE.84.046714

PACS number(s): 05.10.-a, 61.46.Bc, 64.70.Nd, 65.80.-g

\section{INTRODUCTION}

Atomistic modeling of the growth of thin films or selfassembled monolayers has attracted much attention over recent years due to the large number of technological applications in the field of nanotechnology [1-3]. Usually these models include a description of the interaction field between the atoms of the differing species in terms of a potential energy function. The deposition simulation then proceeds either by the addition of another species onto the surface or as a diffusive process where atoms rearrange themselves on the surface. Classical molecular dynamics simulations to model the growth process have been carried out, but usually these involve unrealistically fast deposition rates because the diffusive processes are dominated by rare events and would therefore require extremely long computing times beyond the scope of present computers. Kinetic Monte Carlo (KMC) simulations of the growth processes have also been performed, but in many cases this has required a pre-knowledge of the likely transitions and their barriers since to calculate these on-the-fly has also been a computationally expensive process. Recently, however, some progress has been made into the problem. For example Sprague et al. [4] have shown by using temperature-accelerated dynamics that surface growth patterns are completely different than those that would arise without a proper description of surface diffusion. Other authors have also begun to study the growth process by KMC where the transition barriers are calculated on-the-fly [5-7]. There are, however, still many unsolved problems, especially when the potential energy surface is such that there are many small transition barriers that correspond only to atomic vibration

\footnotetext{
*s.numazawa@hzdr.de

${ }^{\dagger}$ Permanent address: Department of Mathematical Sciences, Loughborough University, Loughborough Le11 3TU, UK.
}

rather than a transition to a new state. In addition, calculating barriers on-the-fly is computationally expensive.

However, even when a traditional KMC process [8] is driven by a preconstructed events table [9], simulations can be inordinately long when there are some transitions that are governed by very small energy barriers but where the main processes that drive the system have much higher barriers. A key aim of the paper is therefore to develop a KMC method that will allow the main escape paths and rate constants to be determined quickly but at the same time will capture the essential physics.

A long-running experimental research project at the HZDR has been in the production of semiconductor crystal surfaces with ripple structures that have a well-defined wavelength [10]. These are produced by oblique incidence bombardment by ion beams and can be controlled by varying the beam angle and energy. It has also been observed that when metal atoms are deposited onto these surfaces the atoms aggregate on the crest of the ripples. A second aim of the paper is therefore to apply the new KMC method for atomistic process simulations where diffusive rather than ballistic processes dominate, such as in this metallic nanocluster growth problem. This will allow a direct comparison between simulation and experimental observations of deposition on ripple structures and therefore help explain the fundamental physical processes that drive the metallic film growth.

To develop the methodology, the general concept of atomistic evolution induced by thermally activated kinetics in a discrete space is considered. A new calculation method including an alternative algorithm for a lattice-based KMC simulation is presented and applied to the problem of nanocluster growth on rippled surfaces. The basic idea of the simulation model is the classification of various transitions into unstable, metastable, and stable (no) transition levels with their transition probabilities depending on local configurations. Under the assumption that metastable transitions dominate the 
entire system evolution, the optimization of their efficiency provides a method to predict the statistical behavior over an extremely long time. This approach can be represented with an oriented graph, considered as an extended percolation model of Fortuin and Kasteleyn [11]. The transition states of cluster growth evolution induces oriented edges, which essentially differ from atomistic interactions based on their application to the Ashkin-Teller-Potts model [12].

The paper is laid out as follows. In Sec. II some terminologies of the mathematical description of the model utilized in this work are defined. In Sec. III every transition event is classified into two types, the first caused by an external factor and the second by a thermal fluctuation. The evolution process induced by the latter type is then localized for the use with the KMC simulation. Section IV discusses the efficiency of the local transition events to the entire system evolution by the introduction of the equivalence class of the fluctuation. The implementation of the modified KMC algorithm is also described. A concrete application to physical vapor deposition (PVD) experiments is presented in Sec. V, where the basic physical growth mechanisms and the stability of the formation process are also discussed.

\section{DEFINITIONS}

In this section several basic terminologies are given.

Let $\mathbb{L}:=\mathbb{Z}_{n_{x}} \times \mathbb{Z}_{n_{y}} \times \mathbb{Z}_{n_{z}}$ be a three-dimensional (3D) lattice space with the spatial periodicity $\left(n_{x}, n_{y}, n_{z}\right)$ for $n_{x}, n_{y}, n_{z} \in \mathbb{N} \cup\{\infty\}$. We define an object $\Omega$ on $\mathbb{L}$ as a Boolean-valued function. A $q$-colored object $\Omega$ is a $q+$ 1 -valued function on $\mathbb{L}$, i.e., all mappings of $\mathbb{L} \rightarrow\{0,1, \ldots, q\}$. The number of colors $q$ corresponds to the number of materials treated in the target simulation. The set of all objects on $\mathbb{L}$ is denoted by $O(\mathbb{L})$. For $\Omega \in O(\mathbb{L}),|\Omega|$ denotes the number of cells occupied by atoms.

A morphism $f$ of $O(\mathbb{L})$ is a mapping from $O(\mathbb{L})$ to itself.

For $\Omega, \Omega^{\prime} \in O(\mathbb{L})$ an ordered pair $\left(\Omega, \Omega^{\prime}\right)$ is called a transition event from $\Omega$ to $\Omega^{\prime}$ and denoted also in the text by $\Omega \rightarrow \Omega^{\prime}$.

The composition of morphisms $f_{m} \circ f_{m-1} \circ \ldots \circ f_{0} \circ \Omega$ on $\mathbb{L}$ induces the Markov chain of objects $\Omega_{0} \rightarrow \Omega_{1} \rightarrow \cdots \rightarrow$ $\Omega_{m}$, where $\Omega_{m^{\prime}}=f_{m^{\prime}} \circ \cdots \circ f_{0} \circ \Omega_{0}$ for $m^{\prime}=0, \ldots, m$. For a Markov chain of objects $\mathcal{M} \equiv \Omega_{0} \rightarrow \cdots \rightarrow \Omega_{m},|\mathcal{M}|$ denotes the length $m$ of the chain.

\section{TRANSITION STATES OF MARKOV CHAINS}

\section{A. Local transition events}

In an atomistic simulation of a solid, the system evolves either through a modification induced by external factors, e.g., a deposition event, through internal dynamical processes that occur more quickly than thermal fluctuations at a constant temperature $T$, or by internal rare events that occur over longer time scales. It is these internal rare events that will be the focus of the work here; i.e., every internal transition event $\left(\Omega_{l}, \Omega_{l+1}\right)$ is temporally well separated, and each microstate of objects is regarded as a quasi-equilibrium state at $T$.

We assume that the transition $\Omega_{l} \rightarrow \Omega_{l+1}$ has the unique decomposition into the elementary transition states induced by an atomic jump attempt including exchange
$\Omega_{l}=v_{0} \rightarrow \cdots \rightarrow v_{s^{\prime}}=\Omega_{l+1}$. These jump events are assumed to occur locally; i.e., the interaction distance is restricted so that an atomic jump occurs only to the nearest-, or second nearest-, neighbor position. Let $\wp_{\text {loc }}^{*}$ be the set of all morphisms representing such events. The transition events $\nu \rightarrow \mu$ of the objects $v, \mu \in O(\mathbb{L})$ induced by $f \in \wp_{\text {loc }}^{*}$ will be termed local transition events, and the decomposition of the transition event $\left(\Omega, \Omega^{\prime}\right)$ into the local transition event together with the sequence of jump attempts will be called the local decomposition of $\left(\Omega, \Omega^{\prime}\right)$.

\section{B. The Monte Carlo method}

Let $\Omega_{l} \rightarrow \cdots \rightarrow \Omega_{l^{\prime}}$ be a subchain of events lying between two external modification events. Consider its local decomposition $\Omega_{l}=v_{0} \rightarrow v_{1} \rightarrow \cdots \rightarrow v_{s}=\Omega_{l^{\prime}}$ and set this Markov chain as $\mathcal{M}$. The total length of the decomposition is

$$
s=\sum_{t=l}^{l^{\prime}-1}\left|\Omega_{t}\right| .
$$

Now consider simulating this Markov process $\mathcal{M}$ with a Monte Carlo (MC) method. In order to follow the process $\mathcal{M}$ the algorithm is constructed as follows: (1) for every object $v_{j}$ choose an arbitrary morphism $f^{j} \in \wp_{\text {loc }}^{*}$, (2) each local transition event $\left(v_{j}, f^{j} \circ v_{j}\right)$ is considered as a jump attempt and accepted with probability $P\left(v_{j}, f^{j}\right)$, and (3) after each local transition event a local time consumption, e.g., for an atom $i, \tau_{j}(i)=1 /\left|v_{j}\right|$ is added to the MC simulation time. If the atom $i$ jumps to a nonequilibrium state, then the jump attempt continues until it reaches a position considered as an equilibrium state without adding $\tau_{j}$.

The process (3) implies that every transition is considered as the transition between two equilibrium states that are not always consistent with the nearest lattice positions. Note that one iteration of the simulation step (1)-(3) advances the MC simulation time only $\tau_{j}$ time units, and it continues for $j=$ $0, \ldots, s-1$.

Let $n$ be the number of total types of events, and for $k=1, \ldots, n$, let $\alpha_{k}$ be the acceptance number of the type $k$ local transition event in $\mathcal{M}$. Then the $n$-events acceptance distribution $\left(\alpha_{k}\right):=\left(\alpha_{1}, \ldots, \alpha_{n}\right)$ models the evolution of the system. Since the local transition probability $P\left(v_{j}, f^{j}\right)$ is a functional of the object $v_{j}$ and the morphism $f^{j}$ that decide the migration barrier, $P$ is also a function of the migration barrier and categorized into $n$ types, $P_{1}, \ldots, P_{n}$. Without lost of generality, it is assumed that $P_{1} \geqslant P_{2} \geqslant \cdots \geqslant P_{n}$. Then the length $s$ of the Markov chain $\mathcal{M}$ is approximately

$$
s \approx \sum_{k=1}^{n} \alpha_{k} P_{k}^{-1},
$$

and the total MC simulation time unit $t_{\mathrm{MCT}}^{\mathcal{M}}$ is

$$
t_{\mathrm{MCT}}^{\mathcal{M}}=l^{\prime}-l
$$

The process of $\mathcal{M}$ corresponds to the experimental evolution time and can be extremely long and impractical to evaluate within reasonable computation time. In the following section we consider the effective reduction of this process. 


\section{EFFECTIVE TRANSITIONS OF OBJECTS}

In this section, the relation between the accepted event distribution $\left(\alpha_{k}\right)$ defined in Sec. III B and the fluctuation of the potential energy sequence is considered. This leads to a method to reduce the number of simulation steps. The effect of this in terms of the thermal fluctuations is discussed.

\section{A. Convergence of the local fluctuation}

Denote by $\mathcal{M}_{\iota}$ the local decomposition of the subchain $\Omega_{l} \rightarrow \cdots \rightarrow \Omega_{l+l}$ for $\iota=0, \ldots, l^{\prime}-l$ and $\mathcal{M}=\mathcal{M}_{l^{\prime}-l}$ as in Sec. III B. Consider the behavior of the thermal fluctuations of the microcanonical ensemble. Set

$$
\Delta t:=l^{\prime}-l
$$

the time interval of the external modification that is preset in the model, e.g., from experimental data so that

$$
\Delta t=t_{\exp }^{\mathcal{M}} \omega_{\text {eff }}
$$

for an experimental time interval $t_{\exp }^{\mathcal{M}}$ and the average effective transition frequency $\omega_{\text {eff }}$ of the system. Note that the corresponding time interval of every event $\left(\Omega_{\iota^{\prime}}, \Omega_{\iota^{\prime}+1}\right)$ is thus $\omega_{\text {eff }}^{-1}$. Let $\left(\alpha_{k}^{l}\right)$ be the partial distributions of the accepted events distribution in $\mathcal{M}_{l}$. If $\Delta t$ is long compared to the recovery time from an unusual fluctuation induced by the last external modification to a usual fluctuation (quasi-equilibrium state), then the convergence of the acceptance of each event $\alpha_{k}^{l} \rightarrow \alpha_{k}$ for $\iota \rightarrow \Delta t$ is also to be considered. Let $\varepsilon$ be the range of the usual fluctuation of the potential energy of the system at a temperature $T$. Then one finds the minimal value $\iota_{0}=\iota_{0}(\varepsilon)$ with the property that

$$
\left|\Phi_{p}\left(\Omega_{l^{\prime}}\right)-\Phi_{p}\left(\Omega_{l^{\prime \prime}}\right)\right|<\varepsilon
$$

for all $\iota^{\prime}, \iota^{\prime \prime} \in\left[\iota_{0}, \Delta t\right]$, where $\Phi_{p}(\Omega)$ indicates the potential energy of $\Omega$. Denote

$$
\theta(\iota):=\frac{\iota}{\Delta t},
$$

the convergence factor of the subchain $\Omega_{l} \rightarrow \cdots \rightarrow \Omega_{l^{\prime}}$, in the sense of the potential energy transition within the fluctuation range $\varepsilon . \theta\left(\iota_{0}\right)=: \theta_{0}$ is the minimum convergence factor in this sense. Let $\left(\alpha_{k}^{t}\right)$ be the continuum extension with respect to the time parameter $\iota$ of $\left(\alpha_{k}^{l}\right)$. Then the dissipation of unusual fluctuations implies $\partial_{t} \alpha_{k}^{t}=0$ for $t>\iota_{0}$ and for usual fluctuations $\partial_{t} \alpha_{k}^{t} \neq 0$. The set of microstates consistent with the fluctuating system is the set of all accessible states with the screened transition types, i.e.,

$$
\left\{\Omega_{\iota}: \iota \in\left[\iota_{0}, \Delta t\right]\right\}=\left[\Omega_{\iota_{0}}\right],
$$

where $\left[\Omega_{\iota_{0}}\right]$ is the equivalence class of objects connected by the pathways consisting of type $k$ transitions with $\partial_{t} \alpha_{k}^{t} \neq 0$ for $t>$ $\iota_{0}$. These transitions are small barriers, and the introduction of an equivalence class for the whole process is the core idea of the modified MC algorithm described in Sec. IV C. Due to the principle of equal a priori probabilities, every microstate belonging to the class $\left[\Omega_{\iota_{0}}\right]$ appears with same probability in the sequence $\Omega_{\iota_{0}} \rightarrow \cdots \rightarrow \Omega_{l^{\prime}}$. Consequently, it is enough to consider the Markov chain $\mathcal{M}_{\iota_{0}}$ to follow the essential system evolution. The convergence factor (6) indicates how the physical process is shortened.
Since the number of transition attempts, i.e., the effective jump frequency, is independent of the system evolution, the length of the local decomposition $\mathcal{M}_{\iota}$ is approximately

$$
\left|\mathcal{M}_{\iota}\right| \approx \theta(\iota) s=\frac{\iota s}{t_{\mathrm{MCT}}^{\mathcal{M}}}
$$

from Eqs. (3), (4), and (6)

\section{B. The importance of individual local transitions}

The contribution of each local transition event to the entire system evolution is not straightforward. Clearly, for a high transition probability $P_{k}$ a large number of $\alpha_{k}$ is expected. However, the number of accepted jumps also depends on the frequency of occurrence of the configuration. The frequency of occurrence distribution can be represented as

$$
\left(\beta_{k}\right):=\left(\alpha_{1} / P_{1}, \ldots, \alpha_{n} / P_{n}\right) .
$$

The quantity $\beta_{k}$ is called the attempt frequency of the type $k$ event. The relation $\beta_{k}<\beta_{k^{\prime}}$ implies that the configuration giving the type $k^{\prime}$ transition is more preferred than the type $k$ transition event. An excessive acceptance of certain types of event does not always mean a change. For example, an adatom deposited on a flat crystal surface can diffuse around until it reaches a defect or a step edge. During diffusion, the correlation between the deposition point and the current position is lost, and only when the adatom finally reaches a binding site does the system evolution proceed further. The crucial evolution of the system occurs with a metastable state transition such as overcoming a step-edge barrier. The model should ensure that the total potential energy does not change substantially through the reduction in the number of $\alpha_{k}$ s that the evolution of system is misdirected.

The activation energy barrier border between the unstable (usual fluctuations) and metastable states is defined to be as the boost energy $E_{\mathrm{bst}}$, and all activation energies above $E_{\mathrm{bst}}$ are considered as effective migration barriers contributing to unusual fluctuations. The boost energy is a parameter that needs to be carefully set for each simulation so that the dynamics is not distorted by choice of too high a factor. Let $P_{n_{0}}$ be the transition probability induced by the smallest effective migration barrier. Now consider the identification of transitions with small fluctuations. From the mathematical point of view, this coarse graining introduces the equivalence relation in the set of all objects $v, \mu \in \mathcal{O}(\mathbb{L})$ as follows:

$$
\begin{gathered}
v \sim \mu \Leftrightarrow \quad \exists a \in \mathbb{N}, \exists f_{1}, \ldots, f_{a} \in \wp_{\mathrm{loc}}^{*}, \\
\mu=f_{a 0} \circ v, \\
\text { with } \quad P\left(f_{a^{\prime}-10} \circ v, f_{a^{\prime}}\right)>P_{n_{0}} \\
\text { for } \quad a^{\prime}=1, \ldots, a .
\end{gathered}
$$

The corresponding equivalence class of objects is $[\nu]$ in $O(\mathbb{L})$. In the worst case, namely, for a system with a high entropy, each equivalence class retains all possible configurations consisting of identical atoms. However, if high potential barriers separate the states, then this enhances the effect of the metastable transitions.

By introducing this classification method the acceptance of the local transition probability with small barriers is 
maximized and the time spent to distinguish the local transition events with small barriers is considerably reduced.

\section{The modified algorithm for effective transition states}

The modification $P_{k}^{\prime}$ of the transition probability $P_{k}$ for the type $k$ local jump attempt described in Sec. IVB is implemented as

$$
P_{k}^{\prime}= \begin{cases}1 & \left(k<n_{0}\right) \\ P_{k} / P_{\mathrm{bst}} & (\text { otherwise })\end{cases}
$$

for the boost probability $P_{\mathrm{bst}}$ corresponding to the acceptance of the highest barrier $E_{\mathrm{bst}}$ within the usual fluctuations. The local time consumption $\tau^{\prime}(i)$ of this type $k$ transition attempt of atom $i$ is set as

$$
\tau^{\prime}(i)= \begin{cases}0 & \left(k<n_{0}\right) \\ \tau(i) & \text { (otherwise) }\end{cases}
$$

So every simulation step that is spent for the transition with barrier less than $E_{\mathrm{bst}}$ does not advance the time step.

Now consider a KMC simulation with the algorithm mentioned above. Let $\mathcal{M}^{\prime} \equiv \Omega_{l}=v_{0} \rightarrow v_{1}^{\prime} \rightarrow \cdots \rightarrow v_{s^{\prime}}^{\prime}$ be the Markov chain describing the simulation consisting of the local transition events with the modified acceptance probability (9). Assume $\mathcal{M}^{\prime}$ reproduces the experimentally observed evolution process and let $\left(\alpha_{k}^{\prime}\right):=\left(\alpha_{n_{0}}^{\prime}, \ldots, \alpha_{n}^{\prime}\right)$ be the corresponding accepted event distribution. If the system evolution excludes this probability translation, then the number of accepted events for the effective transitions should be approximately the same as the number of those transitions in $\mathcal{M}_{\iota}$ for some $\iota \geqslant \iota_{0}$, i.e.,

$$
\alpha_{k}^{\iota} \approx \alpha_{k}^{\prime} \quad \text { for } \quad k=n_{0}, \ldots, n
$$

It is important that the system evolution proceeds according to the property (11).

The translation mapping (9) enhances the acceptance of effective transitions linearly while one might expect a continuous dynamical evolution would induce a nonlinear enhancement. This also affects the attempt frequency (8) of effective transitions, from preferred local configurations. If various metastable transition events determine the system evolution, then the relative acceptance ratio plays a crucial role in the formation of local configurations, and a nonlinear translation of $\left(P_{k}\right)$ would therefore have a high risk of losing the correct evolution of the system since it does not keep same relative acceptance ratio.

For the Markov chain $\mathcal{M}_{\iota}$, the length is $\theta(\iota) s$ from Eq. (7) and can be represented as

$$
\theta(\iota) s=\delta_{\mathrm{bst}}+\sum_{k=n_{0}}^{n} \alpha_{k}^{\iota} P_{k}^{-1}
$$

from the same argument by (2), where $\delta_{\text {bst }}$ is the number of simulation steps spent for local transition events with small barriers $\leqslant E_{\mathrm{bst}}$. Since the translation mapping for the effective transition events is linear, a reduction in the time required for the MC simulation with the boost factor $P_{\mathrm{bst}}^{-1}$ is expected. The total length of $\mathcal{M}^{\prime}$, describing the simulation system evolution, is

$$
s^{\prime}:=\delta_{\mathrm{bst}}^{\prime}+P_{\mathrm{bst}} \sum_{k=n_{0}}^{n} \alpha_{k}^{\iota} P_{k}^{-1} .
$$

Hence, if the Markov chain $\mathcal{M}^{\prime}$, i.e., the KMC simulation, reproduces the experimentally observed evolution process with the reduced simulation steps $s^{\prime}$, then the reduction expectancy in the number of simulation steps $\left|\mathcal{M}^{\prime}\right| /|\mathcal{M}|\left(=s^{\prime} / s\right)$ is given by the relation

$$
\frac{s^{\prime}}{s}=\frac{\delta_{\mathrm{bst}}^{\prime}}{s}+P_{\mathrm{bst}}\left(\theta-\frac{\delta_{\mathrm{bst}}}{s}\right),
$$

from Eqs. (12) and (13). Additionally, if the number of total atoms in the system is relatively stable during the original Markov process $\mathcal{M}$, one can estimate the length $|\mathcal{M}|$ as

$$
s \approx t_{\text {exp }}^{\mathcal{M}} \omega_{\text {eff }}\left|\Omega_{l}\right|
$$

from Eqs. (1) and (5). Similarly, since the MC simulation time counts the number of the effective transitions with (10), it follows that

$$
s^{\prime}-\delta_{\mathrm{bst}}^{\prime} \approx t_{\mathrm{MCT}}^{\mathcal{M}^{\prime}}\left|\Omega_{l}\right|
$$

for the MC simulation time $t_{\mathrm{MCT}}^{\mathcal{M}^{\prime}}$ of $\mathcal{M}^{\prime}$. Moreover, the maximization of the small barrier transition acceptance (9) implies

$$
\frac{\delta_{\mathrm{bst}}}{s}<\theta(\iota) \frac{\delta_{\mathrm{bst}}^{\prime}}{s^{\prime}} .
$$

Thus one can evaluate the convergence factor $\theta$ with Eqs. (14) (17) and the simulation value $s^{\prime}, \delta_{\mathrm{bst}}^{\prime}$ as

$$
\frac{t_{\mathrm{MCT}}^{\mathcal{M}^{\prime}}}{t_{\mathrm{exp}}^{\mathcal{M}} \omega_{\mathrm{eff}} P_{\mathrm{bst}}}<\theta(\iota)<\frac{t_{\mathrm{MCT}}^{\mathcal{M}^{\prime}}}{t_{\mathrm{exp}}^{\mathcal{M}} \omega_{\mathrm{eff}} P_{\mathrm{bst}}}\left(1-\frac{\delta_{\mathrm{bst}}^{\prime}}{s^{\prime}}\right)^{-1} .
$$

The relation (18) indicates the stability range in the sense of the evolution speed of the system that can be reproduced by the consistent modified KMC simulation $\mathcal{M}^{\prime}$. In other words, a successful reproduction by simulation $\mathcal{M}^{\prime}$ suggests that the convergence factor $\theta(\iota)$ is in the range of (18) and $\theta(\iota)>\theta_{0}$. For the minimum Markov chain $\mathcal{M}_{\iota_{0}}$ and the convergence factor $\theta_{0}$, the minimum KMC time range required is from (18)

$$
t_{\mathrm{exp}}^{\mathcal{M}} \omega_{\mathrm{eff}} P_{\mathrm{bst}} \theta_{0}\left(1-\frac{\delta_{\mathrm{bst}}^{\prime}}{s^{\prime}}\right)<t_{\mathrm{MCT}}^{\mathcal{M}^{\prime}}<t_{\exp }^{\mathcal{M}} \omega_{\mathrm{eff}} P_{\mathrm{bst}} \theta_{0} .
$$

Thus the possible reduction rate of simulation time is from (3), (4), and (5) and this argument:

$$
\frac{t_{\mathrm{MCT}}^{\mathcal{M}^{\prime}}}{t_{\mathrm{MCT}}^{\mathcal{M}}} \sim P_{\mathrm{bst}} \theta_{0} .
$$

Note that if $P_{\mathrm{bst}}$ is chosen so small that $E_{\mathrm{bst}}$ exceeds the usual fluctuation range of the system, the simulation cannot follow the evolution process accurately.

\section{CLUSTER GROWTH SIMULATION MODELS}

Although the method described in the previous sections is general to many lattice-based systems, a strong motivating factor for its development was an understanding of experimental work of Ranjan et al. [13], concerned with the deposition of 
$\mathrm{Au}$ and Ag clusters on silica surfaces where ripple structures had been prepatterned using an ion beam. In this section the application to this problem is described. We have also tested the method on some simple one-dimensional examples where a full KMC simulation without a time boost was also undertaken. This confirmed that the results of the full and boosted method were almost equivalent within a certain threshold of the boost factor [14]. For Ag and Au deposition application, however, a full $\mathrm{KMC}$ simulation is too costly.

The simulation system is a simple cubic lattice of size $512 \times$ $512 \times 128$ with the periodic boundary conditions applied in the plane of the surface. The diagonal length of each square lattice cell is the equilibrium interatomic distance of $\mathrm{Au}$ and $\mathrm{Ag}$ in the perfect fcc crystal structure, i.e., 1 lattice unit (lu) corresponds to $0.204 \mathrm{~nm}$ for the both cases. The model assumes that depositing and diffusing metal atoms are only allowed to occupy fcc sites within the perfect cubic overlayer, so grain boundaries are excluded.

The initial surface is shown in Fig. 1(a) with three clearly defined ripple peaks along with the direction at which the $\mathrm{Ag}$ atoms deposit. The ripple shape in Fig. 1(a) is an excellent approximation to the cross-sectional transmission electron microscope image of the $\mathrm{SiO}_{2}$ substrate [Fig. 1(b)]. The asymmetrical ripple surface shown in Fig. 1(a) is set as $\Omega_{0}$ and held fixed during the simulations. This substrate is also chosen to be commensurate with the metal crystal overlayer. $\mathrm{SiO}_{2}$ is regarded as an homogeneous material and therefore $q=2$ in $O(\mathbb{L})$, namely, the metallic atoms are regarded as the type I atoms while every $\mathrm{SiO}_{2}$ component is type II.

The internal modification of the system (local transition morphisms $\wp_{\text {loc }}^{*}$ ) is handled by single atomistic jumps of type I atoms to the nearest-neighbor (NN) empty positions as the local transition. Concerted motions are not allowed. The external modification to the system occurs through the deposition of metal monomers. The depositing atoms are simply set in the positions exposed to the incident trajectories,

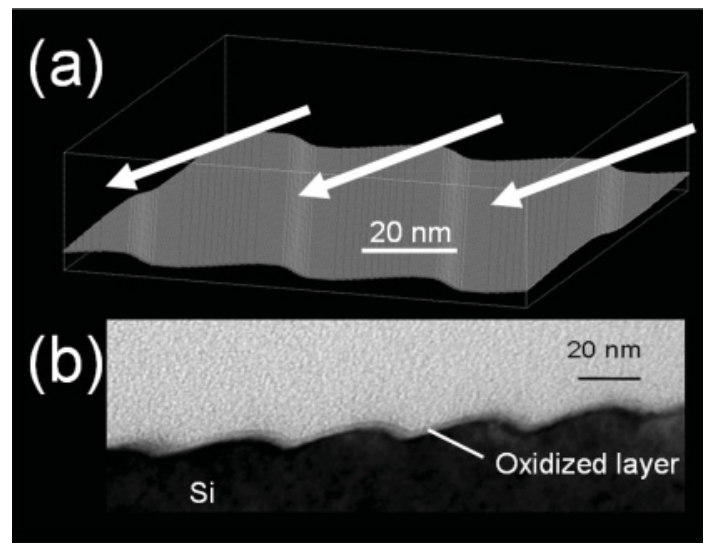

FIG. 1. (a) The initial surface of the simulation model, together with the direction of the arriving flux from the evaporation source (perpendicular to the ripple structures). The asymmetrical ripple shape is fitted to represent the rippled $v-\mathrm{SiO}_{2}$ surface shown in (b). (b) A typical cross-TEM image of rippled Si substrate. The surface layers $(\sim 2 \mathrm{~nm})$ are oxidized by exposure to the natural environment [15]. The wavelength of the ripples is $33 \mathrm{~nm}$ and the height of the ripples is $2.4 \mathrm{~nm}$. and neither reflection nor surface drifting on impact is considered. The atoms detaching from metallic clusters or the substrate are regarded as re-evaporated atoms and eliminated from the simulation. Thus there is no effect of redeposition of atoms returning to the gas phase.

The KMC simulation proceeds either by a single deposition event on to the surface or through the jump of a surface atom. The relative frequency of a deposition event is chosen to match experimental flux rates, but the acceptance or otherwise of a candidate jump event is determined from energies obtained from the geometrical arrangements and types of the neighboring sites. The way in which these energies are determined is given in the next section.

\section{A. The configuration energy and migration barriers}

In order to compute barriers quickly while maintaining the important physics of the system a combination of a relatively simple many-body potential energy function is chosen, the Rosato-Guillope-Legrand (RGL) potential [16], together with migration values taken from the literature. In the description that follows the same symbols are used for the potential parameters as in the original paper. For a type I atom $i$, let $n_{i}^{\mathrm{I}}$ and $n_{i}^{\mathrm{II}}$ be the number of type I, and type II NN atoms, respectively. The potential energy $E_{i}$ of $i$ is defined by

$$
E_{i}=-\zeta \sqrt{n_{i}^{\mathrm{I}}}+A n_{i}^{\mathrm{I}}-E_{i}^{\mathrm{M}-\mathrm{SiO}_{2}}\left(n_{i}^{\mathrm{II}}\right)
$$

where the first two terms are determined from the RGL potential with parameters $\zeta=1.8352 \mathrm{eV}(\mathrm{Au}), 1.1663 \mathrm{eV}$ (Ag), $A=0.2179 \mathrm{eV}$ (Au), $0.09982 \mathrm{eV}$ (Ag) [17], and $E_{i}^{\mathrm{M}-\mathrm{SiO}_{2}}\left(n_{i}^{\mathrm{II}}\right)$ is the metal-SiO${ }_{2}$ interaction term, which is given by

$$
E_{i}^{\mathrm{M}-\mathrm{SiO}_{2}}\left(n_{i}^{\mathrm{II}}\right)= \begin{cases}0 & \left(n_{i}^{\mathrm{II}}=0\right) \\ E_{b}^{\mathrm{M}-\mathrm{SiO}_{2}} & \text { (otherwise) }\end{cases}
$$

The potential functional $\Phi_{p}$ is defined as

$$
\Phi_{p}(\Omega)=\sum_{i} E_{i}
$$

where $i$ ranges all type I atoms in $\Omega \in O(\mathbb{L})$. Let $v \in O[\mathbb{L}]$ be an object. For two sites $u_{\text {ini }}, u_{\text {fin }} \in \mathbb{L}$, let $u_{\text {ini }}$ be the positions where the atom $i$ is located and $u_{\text {fin }}$ be an empty NN position of $u_{\text {ini }}$ represented by $v$, i.e., $\left|u_{\text {ini }}-u_{\text {fin }}\right|=\sqrt{2}[\mathrm{lu}], v\left(u_{\text {ini }}\right)=$ 1 , and $v\left(u_{\text {fin }}\right)=0$. For a local transition morphism $f:=$ $f_{j}\left(1, u_{\text {ini }}, u_{\text {fin }}\right)$, set $v^{\prime}:=f \circ v$. Then the migration barrier $E_{m}(\nu, f)$ is given by

$$
E_{m}(v, f)= \begin{cases}0 & {\left[\Phi_{p}(v)>\Phi_{p}\left(v^{\prime}\right)\right]} \\ \Phi_{p}\left(v^{\prime}\right)-\Phi_{p}(v) & {\left[\Phi_{p}(v)<\Phi_{p}\left(v^{\prime}\right)\right],} \\ E_{\mathrm{mig}}\left(v, v^{\prime}\right) & {\left[\Phi_{p}(\nu)=\Phi_{p}\left(v^{\prime}\right)\right]}\end{cases}
$$

where $E_{\text {mig }}$ is the migration barrier list for potential energy conservative transitions determined from literature values and described in more detail below. The advantage of this functional (22) is to give a quick estimation of the migration barriers such as the Ehrlich-Schwoebel (ES) barrier and various surface migration barriers during the atomistic movement along the 
network of fcc sites. In the first case of (22), the model assumes that the transition barrier is negligible if the final configuration $v^{\prime}$ is more stable than the initial configuration $v$. Particularly it allows atoms overcoming the ES barrier to move freely until they reach stable positions. This could, however, cause an error if the initial configuration $v$ is already a quasi-equilibrium state.

Since the transition event modifies only the local configuration around the jumping atom, the potential energy change occurs only for the atoms neighboring the initial site and the final site of the jumping atom. These initial and final positions are surrounded by $18 \mathrm{NN}$ sites and 52 other sites whose potential energy is affected by the transition. Hence the total number of possible configurations are $3^{70}$ (70 sites and three possibilities at each site, i.e., empty, type I atom, or type II atom). However for the efficiency of the calculation, only the $18 \mathrm{NN}$ positions surrounding the initial and the final sites are used to determine the migration energy.

Figure 2 shows $u_{\text {ini }}, u_{\text {fin }}$ and the $18 \mathrm{NN}$ positions that are divided into the sets $N_{i}:=\{1,2,3,4,5,6,11\}, N_{c}:=$ $\{7,9,10,12\}$ and $N_{f}:=\{8,13,14,15,16,17,18\}$ with respect to the neighboring $u_{\text {ini }}$ and $u_{\text {fin }}$. The positions belonging to $N_{i}$ and $N_{f}$ are NN sites of the initial site $u_{\text {ini }}$ only, and of the final site $u_{\text {fin }}$ only, respectively. The positions of $N_{c}$ are the common NNs of the both sites. Since the configuration energy of each atom is calculated by (20), the local transition morphism $\wp_{\text {loc }}^{*}$ consists of all transition types with the form $\left(\left|N_{i}^{\mathrm{I}}\right|,\left|N_{i}^{\mathrm{II}}\right|,\left|N_{c}^{\mathrm{I}}\right|,\left|N_{c}^{\mathrm{II}}\right|,\left|N_{f}^{\mathrm{I}}\right|,\left|N_{f}^{\mathrm{II}}\right|\right)$, where $\left|N_{*}^{\mathrm{I}, \mathrm{II}}\right|$ indicates the number of occupied NN sites by type-I, as well as type-II atoms belonging to the position index set $N_{*}$. Since the type-II atoms are fixed and occupy at most $6 \mathrm{NN}$ positions, it is possible to show that the maximal number of different 18 NN local configurations with respect to the combination of $\left|N_{i}^{\mathrm{I}}\right|,\left|N_{i}^{\mathrm{II}}\right|,\left|N_{c}^{\mathrm{I}}\right|,\left|N_{c}^{\mathrm{II}}\right|,\left|N_{f}^{\mathrm{I}}\right|$, and $\left|N_{f}^{\mathrm{II}}\right|$ is 1381 . These configurations account for the migration barrier estimation in all cases of jumps between unequal energy sites where the variation of

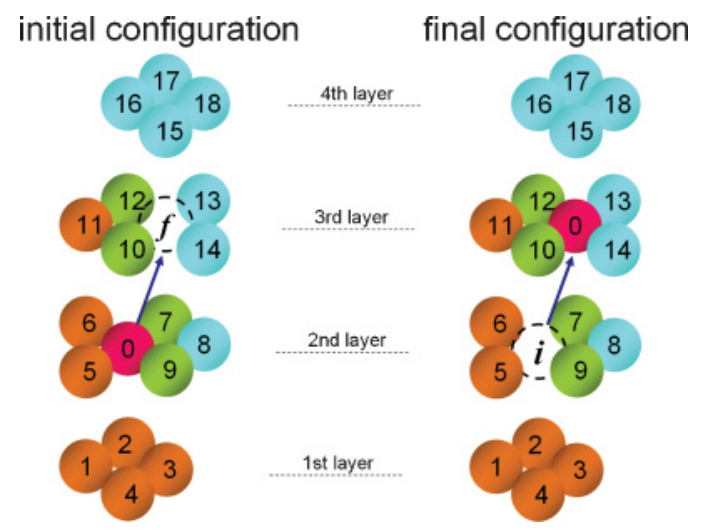

FIG. 2. (Color online) The local NN (fcc) configuration of an initial site $u_{\text {ini }}$ and final site $u_{\text {fin }}$ denoted by $i$ and $f$. For this system, the NN sets for $i$ and $f$ can be seen to be $N_{i}:=\{1,2,3,4,5,6,11\}$ (orange, or dark gray), $N_{c}:=\{7,9,10,12\}$ (green, or gray), and $N_{f}:=$ $\{8,13,14,15,16,17,18\}$ (blue, or light gray). The site 0 is occupied by a Type-I atom, and other sites are either empty, or occupied by a type-I, or a type-II atom. the configuration energy is approximated by

$$
\Phi_{p}(v)-\Phi_{p}\left(v^{\prime}\right) \approx E_{\mathrm{fin}}-E_{\mathrm{ini}},
$$

where $E_{\text {ini }}, E_{\text {fin }}$ are the potential energies before and after a jump as determined by the $18 \mathrm{NN}$ configurations and the expected NN configuration of each neighbor atom. The energy of the initial state $E_{\text {ini }}$ is defined by

$$
E_{\mathrm{ini}}=\sum_{j=0}^{18} \epsilon_{j} E_{j}^{\mathrm{ini}},
$$

where $\epsilon_{j}$ indicates the occupation of the $j$ th site by the type I atom. The configuration energy of each occupying atom is calculated as

$$
E_{j}^{\mathrm{ini}}= \begin{cases}E_{0}^{\mathrm{ini}} & (j=0) \\ E(\bar{n}) & (\text { otherwise }),\end{cases}
$$

where $E_{0}^{\text {ini }}$ is calculated by Eq. (20) for the initial site 0 and $E(\bar{n})$ is the mean configuration energy

$$
E(\bar{n}):=-\zeta \sqrt{\bar{n}}+A \bar{n},
$$

for the other atoms. Here $\bar{n}$ is the average number of type I NNs of all occupied sites by the type I atoms possessing at least one empty NN site. This factor is influenced by the mean surface curvature of clusters growing in the system. The local configuration energy of the final state $E_{\text {fin }}$ is defined similarly as

$$
E_{\mathrm{fin}}=\sum_{j=0}^{18} \epsilon_{j} E_{j}^{\mathrm{fin}},
$$

where the position $j=0$ now implies the final site. Now each $E_{j}^{\mathrm{fin}}$ is given by

$$
E_{j}^{\mathrm{fin}}= \begin{cases}E_{0}^{\mathrm{fin}} & (j=0) \\ E(\bar{n}-1) & \left(j \in N_{i}^{\mathrm{I}}\right) \\ E(\bar{n}) & \left(j \in N_{c}^{\mathrm{I}}\right) \\ E(\bar{n}+1) & \left(j \in N_{f}^{\mathrm{I}}\right) .\end{cases}
$$

The average NN number $\bar{n}$ is updated every 12000 time units.

For the case of the symmetric configurations due to the $\mathrm{NN}$ environment of the initial and the final states where the RGL potential does not change, the migration barrier list $E_{\text {mig }}\left(\nu, v^{\prime}\right)$ shown in Table I is utilized. The cases shown with the zero barriers refer to transitions that are either rare or whose contribution is negligible. The other cases where the initial, final, and common number of nearest neighbors are the same as in the cases of adatom transitions on surfaces, and data from the literature corresponding to these values are used. The transition barriers for the type I atoms are estimated from the adatom migration barriers on (111), (100) and in-channel (110) oriented surfaces calculated in Refs. [18,19]. Although these values are the best estimates for the surface transitions they may be less accurate for some other configurations possessing the same number of $\left|N_{i}^{\mathrm{I}}\right|,\left|N_{f}^{\mathrm{I}}\right|$, and $\left|N_{c}^{\mathrm{I}}\right|$.

Figure 3 shows examples of some configuration energies of atoms located in various positions and a step-edge barrier. Each $E_{i}$ is calculated by Eq. (20) and the step-edge barrier $E_{m}=0.39 \mathrm{eV}$ for the transition indicated is derived for the transition of type $\left(\left|N_{i}^{\mathrm{I}}\right|,\left|N_{c}^{\mathrm{I}}\right|,\left|N_{f}^{\mathrm{I}}\right|\right)=(2,1,1)$, with the average NN site occupation $\bar{n}=8.5$ ( $*$ in Fig. 3 ). 
TABLE I. Migration energy list $E_{\text {mig }}\left(v, v^{\prime}\right)$ for type-I interactions. $\left|N_{*}^{\mathrm{II}}\right|=0$ for every case. The barriers in the table are estimated from the literature adatom migration barriers on the (111), (100) and in-channel (110) oriented surfaces with the same neighbor configurations. $E_{\text {mig }}^{(111)}=0.04,0.07 \mathrm{eV}$ (Ag [18], Au [18]), $E_{\mathrm{mig}}^{(100)}=$ $0.38,0.51 \mathrm{eV}$ (Ag [18], Au [18]), $E_{\text {mig }}^{(110)}=0.28,0.23 \mathrm{eV}$ (Ag [19], Au [18]). The zero barriers correspond to transitions and configurations that have been tested by numerical experiment to be so unimportant that an accurate determination is unnecessary.

\begin{tabular}{ccccccccc}
\hline \hline$\left|N_{i}^{\mathrm{I}}\right|_{\left(=\left|N_{f}^{\mathrm{I}}\right|\right)}$ & 0 & 1 & 2 & 3 & 4 & 5 & 6 & 7 \\
\hline$\left|N_{c}^{\mathrm{I}}\right|=0$ & 0 & 0 & 0 & $E_{\mathrm{mig}}^{(111)}$ & $E_{\mathrm{mig}}^{(100)}$ & $E_{\mathrm{mig}}^{(110)}$ & 0 & 0 \\
$\left|N_{c}^{\mathrm{I}}\right|=1$ & 0 & 0 & $E_{\mathrm{mig}}^{(111)}$ & $E_{\mathrm{mig}}^{(100)}$ & $E_{\mathrm{mig}}^{(110)}$ & 0 & 0 & 0 \\
$\left|N_{c}^{\mathrm{I}}\right|=2$ & 0 & $E_{\mathrm{mig}}^{(111)}$ & $E_{\mathrm{mig}}^{(100)}$ & $E_{\mathrm{mig}}^{(110)}$ & 0 & 0 & 0 & 0 \\
$\left|N_{c}^{\mathrm{I}}\right|=3$ & $E_{\mathrm{mig}}^{(111)}$ & $E_{\mathrm{mig}}^{(100)}$ & $E_{\mathrm{mig}}^{(110)}$ & 0 & 0 & 0 & 0 & 0 \\
$\left|N_{c}^{\mathrm{I}}\right|=4$ & $E_{\mathrm{mig}}^{(100)}$ & $E_{\mathrm{mig}}^{(110)}$ & 0 & 0 & 0 & 0 & 0 & 0 \\
\hline \hline
\end{tabular}

In Fig. 3, the atom $i$ has been deposited on the part of the surface that appears to be an island with a (111) surface orientation, whereas the atom with $E_{i}=-1.93 \mathrm{eV}$ is located on an island with a (100) surface. Compared to all other atoms belonging to the (111) island, the configuration energy of $i$ is quite high. Even for the relatively high-energy atom located along the edge of the island, the reverse migration barrier climbing up to the island top is at least $E_{m}=0.98 \mathrm{eV}$ with the transition type $(3,2,0)$. Such an event is unlikely to happen, and thus the adatom $i$ will be constrained to the island (see also Fig. 8). The migration barrier of $i$ on $\mathrm{Ag}(111)$ is $0.04 \mathrm{eV}$ by Table I and therefore following the scheme outlined in the previous section is regarded as an unusual fluctuation.

The transition probability $P$ for the migration barrier $E_{m}(\nu, f)$ is assumed to follow the Arrhenius law with a constant prefactor for all transitions

$$
P(\nu, f)=\exp \left[-E_{m}(v, f) / k_{B} T\right],
$$

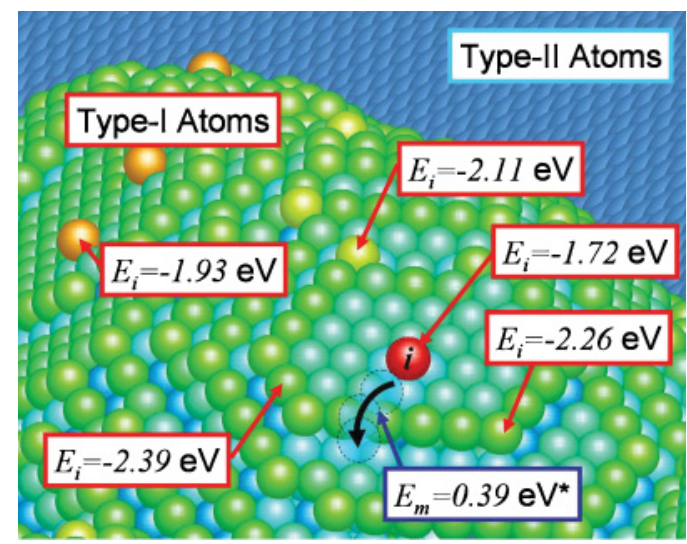

FIG. 3. (Color online) The atomistic landscape during an $\mathrm{Ag}$ PVD simulation: The $E_{i}$ values indicate the individual configuration energies of atoms calculated from Eq. (20). $(*) E_{m}$ is the step-edge barrier for the atom $i$ migrating along the arrow, derived from Eq. (22)-(26) with the average NN site occupation $\bar{n}=8.5$. The color of type-I atoms (online version only) indicates the individual energy states of atoms. where $k_{B}$ is the Boltzmann constant, and the local time consumption for atom $i$ is

$$
\tau(i)=\frac{1}{|\nu|} .
$$

All the sites possessing at least 3 occupied NN sites are considered as equilibrium configurations, and the atom $i$ continues jumping until it gains such a configuration with local time consumption $1 /|\nu|$.

For the initial nucleation distribution, the optimal values $E_{b}^{\mathrm{Ag}-\mathrm{SiO}_{2}}=0.26 \mathrm{eV}, E_{b}^{\mathrm{Au}-\mathrm{SiO}_{2}}=0.24 \mathrm{eV}$ are found during simulations, and it is these energies that are used to determine the lifetime of metal adatoms on the $\mathrm{SiO}_{2}$ surface by (21). A similar adhesion energy range of $\mathrm{Ag}, 0.23 \mathrm{eV}$, on the regular oxide site of $\mathrm{MgO}(100)$ surface is also predicted by the density functional theory calculation [20], whereas a bit higher energy is expected for $\mathrm{Ag}-\mathrm{SiO}_{2}$. The effect of the average adatom lifetime on the nucleation process is described in Ref. [15]. Each transition probability is determined by the primary local configuration with (22) and (27). Thus the entire system evolution between the external modification can be regarded as a class 4 object of a 3D cellular automaton [21].

\section{B. Transition events}

For all positive migration barriers determined by (23) let $E_{m_{1}}, \ldots, E_{m_{n}}$ be the barriers with $E_{m_{1}}<\cdots<E_{m_{n}}$. Set $P_{k}:=$ $\exp \left\{-E_{m_{k}} / k_{B} T\right\}$. For the both PVD simulations the surface transitions

$$
P_{k}^{\prime}:=\exp \left[-\vartheta\left(E_{m_{k}}-E_{\mathrm{bst}}\right) / k_{B} T\right]
$$

are considered with $E_{\mathrm{bst}}=0.20 \mathrm{eV}$. In arriving at this number some simulations were carried out at different $E_{\mathrm{bst}}$ values. With $E_{\mathrm{bst}}=0.30 \mathrm{eV}$, the simulations ran even quicker, but the system evolution is distorted because of the barriers associated with the dominant processes of the migration of metal monomers in the in-channel direction on the (110) oriented surface. These are $0.28 \mathrm{eV}$ for $\mathrm{Ag}$ and $0.23 \mathrm{eV}$ for $\mathrm{Au}$. For a value of $E_{\mathrm{bst}}=0.10 \mathrm{eV}$, the nanostructures showed no observable difference to those formed with $E_{\mathrm{bst}}=$ $0.20 \mathrm{eV}$. Here $\vartheta$ is the temperature factor, which should be close to 1 by the argument in Sec. IV C. Since the PVD experiments were carried out at the room temperature, the temperature parameters are set as $\vartheta=1$ and $T=300 \mathrm{~K}$ during the simulation.

As a result of the choice of $E_{\mathrm{bst}}$, all transition events under a barrier $0.20 \mathrm{eV}$ possess the same acceptance ratio 1. Thus the number of the acceptances for every such event depends only on the frequency of its configuration occurrence. The boost probability $P_{\mathrm{bst}}$, defined in Sec. IVC, is thus $P_{\text {bst }}=\exp \left(-0.2 / k_{B} T\right)=4.37 \times 10^{-4}$, and the boost factor is $P_{\mathrm{bst}}^{-1}=2.29 \times 10^{3}$. Figure 4 shows the list of accepted migration barrier distribution for type I atoms interaction. Every activation barrier is calculated by Eq. (28) and listed in the look-up table. A total of 4039 types (Au) and 4712 types $(\mathrm{Ag})$ local transition events are accepted during $1.2 \times 10^{7}$ time units, corresponding to $3 \mathrm{CPU}$ days' calculation on a typical single-processor desktop machine. However, most of these transition barriers rely on the mean configuration energy term (25), and by fixing the average number $\bar{n}$, the 

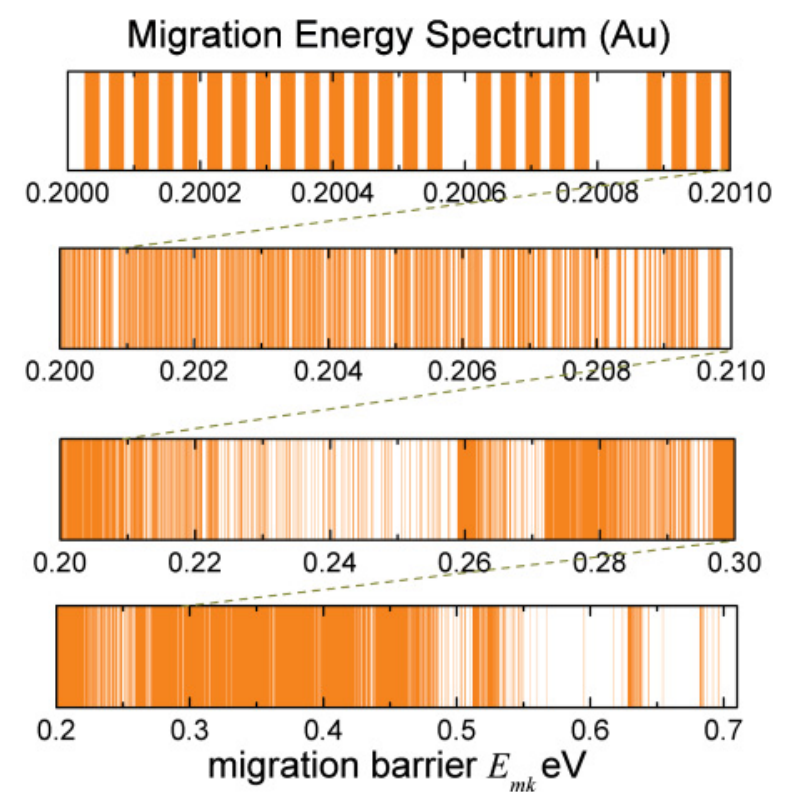

Migration Energy Spectrum (Ag)
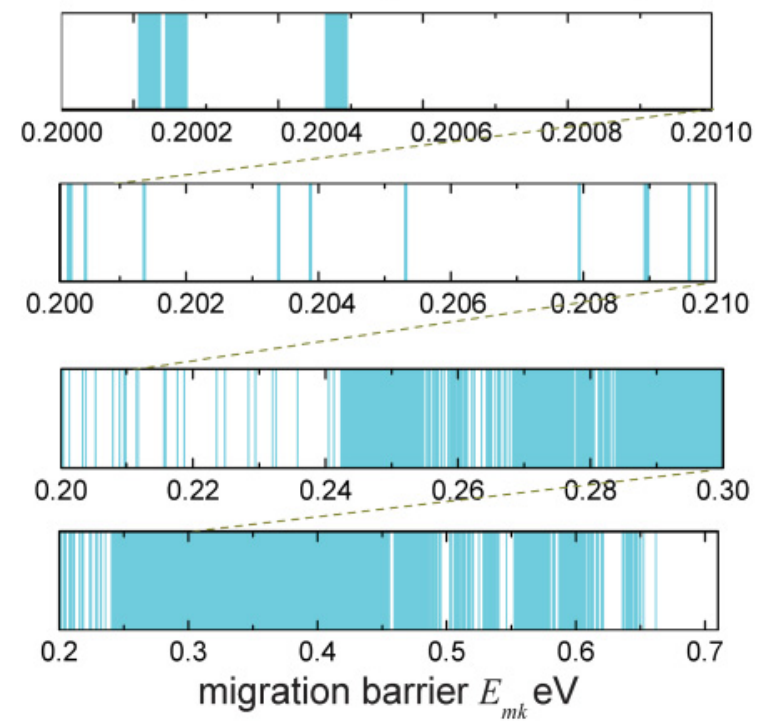

FIG. 4. (Color online) The effective migration barriers estimated by the RGL potential with the parameters [17] for Au and Ag. A total of 4039 types ( $\mathrm{Au}$ ) and 4712 types ( $\mathrm{Ag}$ ) of effective transition events are accepted during a MC simulation time of $1.2 \times 10^{7}$ units.

number of accepted event types is reduced to around 100 . This implies that most transition events have a low probability of acceptance, and only around 100 events represent the metastable transitions. Figure 5 shows the total accepted effective event distribution and its attempt frequency (8) during the simulation of Au and Ag PVD deposition. The distributions $\left(\alpha_{k}\right)$ and $\left(\beta_{k}\right)$ include all local transition events considered as the internal modification activity.

In the $\left(\alpha_{k}\right)$ distribution, the solitary peaks located at $0.23 \mathrm{eV}$, $0.51 \mathrm{eV}(\mathrm{Au})$ and $0.28 \mathrm{eV}$, and $0.38 \mathrm{eV}(\mathrm{Ag})$ correspond to adatom migration attempts on the metal (110) in-channel and (100) surfaces, respectively. The sawlike peaks in the graphs mainly indicate the acceptance of various types of step-edge barriers due to the mean NN configuration. As an $\mathrm{Ag}$ cluster grows, its mean surface curvature decreases. Thus
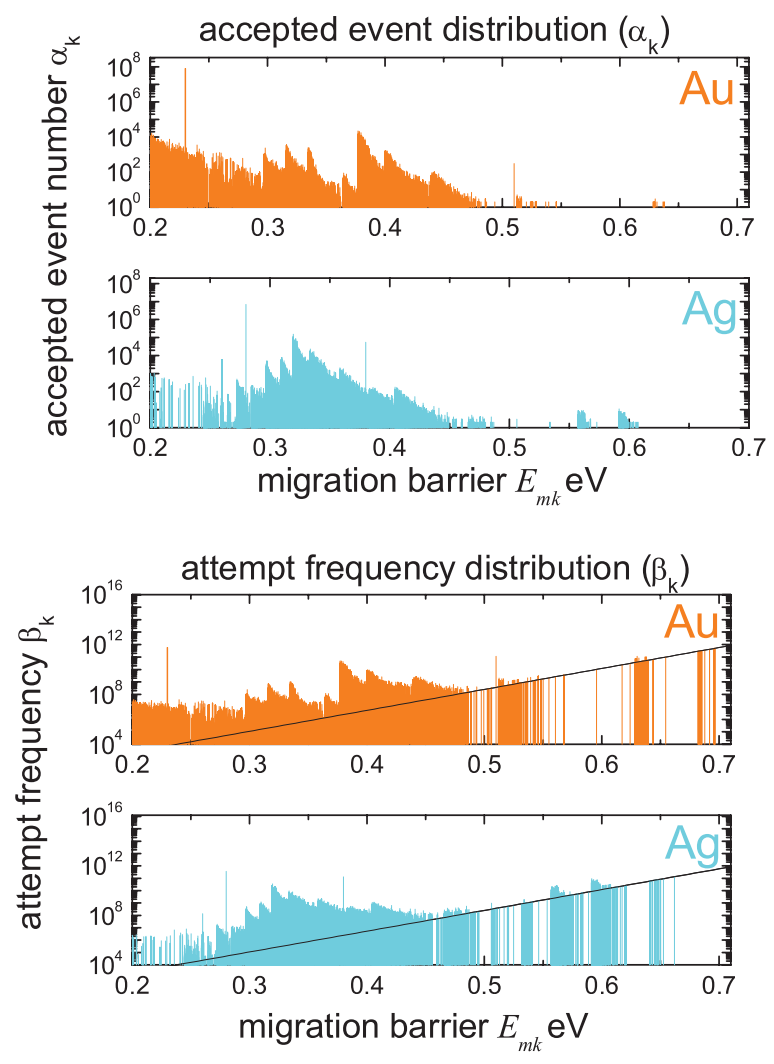

FIG. 5. (Color online) The distribution of accepted events, and the attempt frequencies for the $\mathrm{Au}$ and $\mathrm{Ag}$ growth simulations. Migrations with barriers lying between 0.2 and $0.7 \mathrm{eV}$ are regarded as metastable transition states. The barriers shown are for the interactions of type I atoms only. The solid lines in the $\left(\beta_{k}\right)$ distribution indicate the inverse probability $P_{k}^{-1}$, i.e., the average number of attempts for one acceptance.

the mean value of the $\mathrm{NN}$ occupied positions increases, and consequently, each local transition event reduces the barrier slowly by decreasing the configuration energy difference $E_{j}^{\mathrm{ini}}-E_{j}^{\mathrm{fin}}$ for $j>0$ from Eqs. (24), (25), and (26).

In the $\left(\beta_{k}\right)$ distribution, the attempt frequency for barriers other than the (110) and (100) type transitions slightly increases with increasing barrier height. This indicates that the acceptance of small metastable transitions is already saturated and the system is waiting for a rarer transition with a higher barrier. The solid lines exhibit the average attempt frequency for one acceptance, i.e., $P_{k}^{-1}$, and it can be seen that this average arises from only one transition for the high-barrier events. Hence, as the graph of $\left(\alpha_{k}\right)$ also shows, the acceptance of such a high-barrier event is so small as to have hardly any influence on the system evolution, and thus the practical metastable transition range is anticipated only in the range $0.2-0.6 \mathrm{eV}$.

\section{Comparison with experiment}

Experimentally, the aggregation of clusters is observed mainly where the local flux is a maximum, namely, on the slopes facing the evaporator. A similar tendency is also observed in the simulations. Nucleation occurs when two adatoms migrating on the substrate come together. Due to 
(a)

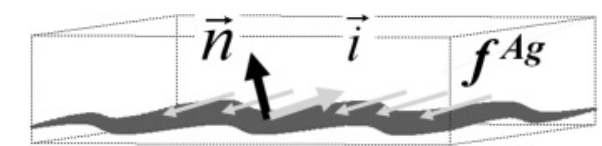

(b)

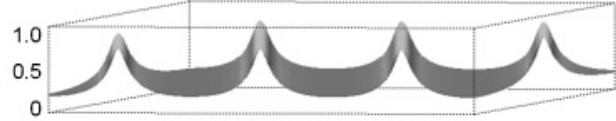

(c)

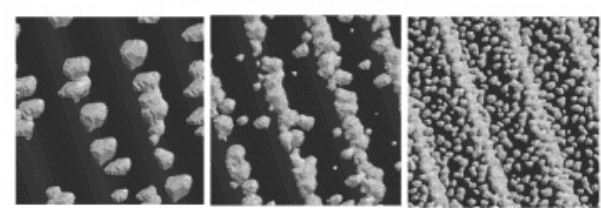

FIG. 6. A schematic description of PVD model and simulation results [15]. (a) The initial rippled surface and schematic description of Ag deposition. Ag flux $f^{\mathrm{Ag}}$, the incident angle vector $\vec{i}$, and the surface normal vector $\vec{n}$ are shown. The angle of incidence in the simulations was fixed at $70^{\circ}$ and perpendicular to the ripple direction. Deposition occurred at a temperature of $300 \mathrm{~K}$. (b) The relative Ag arrival rate $f_{\text {loc }} / f=\vec{n} \cdot \vec{i}$ as a function of surface topography $\vec{n}$ and flux $f \vec{i}$. (c) Three different examples of Ag structures after the equivalent of $6 \mathrm{ML} \mathrm{Ag} \mathrm{deposition.} \mathrm{(Top} \mathrm{view:} 105 \mathrm{~nm} \times 105 \mathrm{~nm}$ ). Left: $E_{b}^{\mathrm{Ag}-\mathrm{SiO}_{2}}=0.245 \mathrm{eV}$; middle: $E_{b}^{\mathrm{Ag}-\mathrm{SiO}_{2}}=0.26 \mathrm{eV}$; and right: $E_{b}^{\mathrm{Ag}-\mathrm{SiO}_{2}}=0.30 \mathrm{eV}$.

the strong binding energy (20) the detachment of a dimer from the surface is unlikely at the simulation temperature of $300 \mathrm{~K}$. However, despite their lower mobility than monomers, a small proportion of dimers still detach from the substrate. The mobility of trimers including detachment is almost negligible. Thus the nucleation probability is strongly influenced by the migration of monomers during the adatom lifetime and the local flux.

In the early stages of growth, nucleation occurs mainly on the slopes facing the incoming $\mathrm{Ag}$ atoms where the local flux is high, but some small clusters are also observed in the lower flux regions where there is also a higher relative detachment rate. Further deposition promotes the Volmer-Weber growth of Ag clusters and their coalescence.

Figure 6 shows a schematic description of metal deposition (a), the relative local flux (b), and three different $6 \mathrm{ML} \mathrm{Ag}$ cluster distribution patterns (c). Because of the initial ripple structure and the $70^{\circ}$ incidence angle, the local flux $f_{\text {loc }}$ is a maximum on the slopes whose normals point toward the evaporation source and minimum on the slopes of the other side [Fig. 6(b)]. In fact $f_{\text {loc }}$ ranges from $20 \%$ to $75 \%$ of the flux $f$ from the evaporator. This strongly localized relative flux rate results in oblique angle deposition on a sawlike asymmetric rippled substrate. The left diagram of Fig. 6(c) is the nucleation pattern for $E_{b}^{\mathrm{Ag}-\mathrm{SiO}_{2}}=0.245 \mathrm{eV}$. For this value of the binding energy, the total sticking probability is only $18 \%$ (i.e., $82 \%$ of deposited atoms evaporate). Arriving atoms rarely stick at first, even in the relatively high local flux regions. Nucleation occurs by $\mathrm{Ag}$ atoms depositing directly onto preformed $\mathrm{Ag}$ clusters, which remain in the system while atoms deposited onto the bare substrate are mainly evaporated. This trend is seen in the Au PVD experiment (see also Fig. 7). The middle and right diagrams show the nucleation pattern when $E_{b}^{\mathrm{Ag}-\mathrm{SiO}_{2}}=0.26 \mathrm{eV}$ and $E_{b}^{\mathrm{Ag}-\mathrm{SiO}_{2}}=0.30 \mathrm{eV}$, respectively. Although these variations in binding energy are quite small,

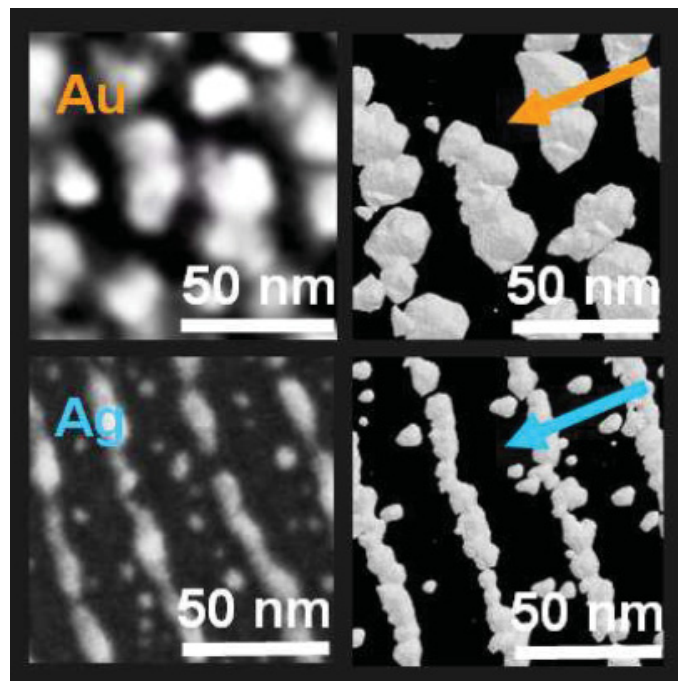

FIG. 7. (Color online) A comparison between the experimental results for Au and Ag PVD with the simulation model. SEM images of the rippled $\mathrm{v}-\mathrm{SiO}_{2}$ surfaces after $\mathrm{Au}$ and $\mathrm{Ag}$ deposition (left) for 30 and 75 minutes, respectively. A top view of the rippled simulation surfaces after $\mathrm{Au}\left(E_{b}^{\mathrm{Au}-\mathrm{SiO}_{2}}=0.24 \mathrm{eV}\right)$ and $\mathrm{Ag}\left(E_{b}^{\mathrm{Ag}-\mathrm{SiO}_{2}}=0.26 \mathrm{eV}\right)$ simulations (right) are shown. It is reported in the literature that the adhesion energy of $\mathrm{Au}-\mathrm{SiO}_{2}$ is less than of $\mathrm{Ag}-\mathrm{SiO}_{2}$ [22]. The total metal accumulation is equivalent to $12 \mathrm{ML}$ for $\mathrm{Au}$ and $6 \mathrm{ML}$ for $\mathrm{Ag}$. Arrows indicate the direction of incident metal atoms.

the adatom lifetime of metal monomers on the bare surface is prolonged exponentially with increasing binding energy. In the middle diagram, nucleation occurs constantly in the high-flux region of the surface, and a coalescence of small $\mathrm{Ag}$ clusters is observed. A few clusters are also observed to grow in the low-flux region. In total $35 \%$ of $\mathrm{Ag}$ atoms remain in the system. Experimentally observed Ag nanowires are quite close to this type of growth mode. In the right diagram, the sticking probability is $82 \%$, and nucleation can occur overall on the substrate surface with the $\mathrm{Ag}$ accumulation following almost the same trend as the local flux distribution of (b).

Figure 7 gives a direct comparison of the simulation results with the SEM images [15,23]. With the chosen binding energy parameters, the simulated metal clusters are very similar to the SEM images. Especially the shape of the cluster edges and sizes agree nicely with the experimental observations. The similarity of the cluster shapes suggests the validity of the chosen parameters for $\mathrm{Au}$ and $\mathrm{Ag}$ and help validate the unusual fluctuation convergence model considered above. The size and coalescence of clusters result in the nucleation probability on the substrate, which is strongly influenced by the adatom lifetime and local flux.

On a (100)-oriented flat substrate, the mean adatom lifetimes of metal monomers $T_{\mathrm{AL}}^{\mathrm{M}}$ with the simulation time unit is given by [15] $T_{\mathrm{AL}}^{\mathrm{M}}=3 \exp \left(E_{b}^{\mathrm{M}-\mathrm{SiO}_{2}}-E_{\mathrm{bst}}\right) / \mathrm{kT}$ units. In the simulation model, $E_{b}^{\mathrm{Au}-\mathrm{SiO}_{2}}=0.24 \mathrm{eV}$ and $E_{b}^{\mathrm{Ag}-\mathrm{SiO}_{2}}=$ $0.26 \mathrm{eV}$ are employed, and therefore the mean adatom lifetime of two metals on flat surface is $T_{\mathrm{AL}}^{\mathrm{Au}}=14.1$ units and $T_{\mathrm{AL}}^{\mathrm{Ag}}=30.6$ units. The total $\mathrm{MC}$ simulation time and the experimental deposition time are $t_{\mathrm{MCT}}^{\text {tot,Au }}=2.7 \times 10^{7}$ units, $t_{\mathrm{MCT}}^{\text {tot, } \mathrm{Ag}}=1.2 \times 10^{7}$ units and $t_{\mathrm{exp}}^{\text {tot,Au }}=1800[\mathrm{~s}], t_{\mathrm{exp}}^{\mathrm{tot}, \mathrm{Ag}}=4500$ 
[s], respectively. Thus to be consistent with experiment, the adatom lifetime of metals is $T_{\mathrm{AL}}^{\mathrm{Au}}=9.40 \times 10^{-4}$ [s] and $T_{\mathrm{AL}}^{\mathrm{Ag}}=1.15 \times 10^{-2}[\mathrm{~s}]$ for Au and Ag, respectively. Hence the simulation model predicts an adatom lifetime of Au monomers that is one-tenth that of $\mathrm{Ag}$ on the bare surface. The consistent MC simulation time units are 1 unit $=6.67 \times 10^{-5}$ [s] for $\mathrm{Au}$ and 1 unit $=3.75 \times 10^{-4}[\mathrm{~s}]$ for Ag, respectively. These film growth rates, assuming unit sticking probability, give flux parameters of $f^{\mathrm{Au}}=0.020\left[\mathrm{ML} \mathrm{s}^{-1}\right]$ and $f^{\mathrm{Ag}}=0.0038$ $\left[\mathrm{ML} \mathrm{s}^{-1}\right]$ and are less than those of Petersen and Mayr [24] by a factor of $O\left(10^{2}\right)$.

\section{Cluster growth stability evaluation}

In order to estimate the stability range (18) of the evolution speed discussed in Sec. IV C, we recall the simulation- and experimental parameters required. In the simulations shown in Fig. 7, $6 \mathrm{ML} \mathrm{Ag} \mathrm{nanocluster} \mathrm{growth} \mathrm{has} \mathrm{been} \mathrm{obtained} \mathrm{after}$ a MC simulation time of $1.2 \times 10^{7}$ units with a boost factor of $2.3 \times 10^{3}$. The experimentally observed Ag nanostructure was grown after 75 minutes of PVD.

The effective transition frequency $\omega_{\text {eff }}$ depends both on the material and the temperature. According to Ref. [25], its range is generally in the region of $10^{12}-10^{13}\left[\mathrm{~s}^{-1}\right]$. Now assume that the effective transition frequency is the Debye frequency $\omega_{\text {eff }}=\omega_{\mathrm{D}}^{\mathrm{M}}$ of the metal to derive the consistent convergence factor evaluated in (18) and (6) with the simulation. The total deposition time of an Ag PVD experiment is $t_{\text {exp }}^{\text {tot }}=4500$ [s]. The total simulation fluence is $F=175$ [atoms $/ \mathrm{nm}^{2}$ ], corresponding to $15 \mathrm{ML}$ of $\mathrm{Ag}$ deposition. The total MC simulation time is $t_{\mathrm{MCT}}^{\mathrm{tot}}=1.2 \times 10^{7}$ units with $3 \%$ of all the transition events belonging to those described as usual fluctuations. The Debye frequency of $\mathrm{Ag}$ is $\omega_{\mathrm{D}}^{\mathrm{Ag}}=2.95 \times 10^{13}\left[\mathrm{~s}^{-1}\right]$.

Let $\mathcal{M}, \mathcal{M}^{\prime}$ be the original and modified simulation process in a typical interval between two Ag depositions in a small region, respectively. Let $t_{\exp }^{\mathcal{M}}, t_{\mathrm{MCT}}^{\mathcal{M}^{\prime}}$ be the experimental, and MC simulation time as defined in Sec. III B and Sec. IV A. Assume the uniform reduction

$$
\frac{t_{\mathrm{MCT}}^{\mathcal{M}^{\prime}}}{t_{\exp }^{\mathcal{M}}} \approx \frac{t_{\mathrm{MCT}}^{\mathrm{tot}}}{t_{\mathrm{exp}}^{\mathrm{tot}}},
$$

then the relation (18) is given by

$$
\frac{t_{\mathrm{MCT}}^{\mathrm{tot}}}{t_{\exp }^{\mathrm{tot}} \omega_{D}^{\mathrm{Ag}} P_{\mathrm{bst}}}<\theta(\iota)<\frac{t_{\mathrm{MCT}}^{\mathrm{tot}} 0.97^{-1}}{t_{\exp }^{\mathrm{tot}} \omega_{D}^{\mathrm{Ag}} P_{\mathrm{bst}}} .
$$

Thus $\theta(\iota)$ is in the range $2.07 \times 10^{-7}<\theta(\iota)<2.13 \times$ $10^{-7}$, and the mean acceleration of the simulation time is $\left[P_{\mathrm{bst}} \theta(\iota)\right]^{-1} \sim 1.09 \times 10^{10}$ from (19). To see the situation more clearly, consider a concrete example. Figure 8 shows the deposition of an $\mathrm{Ag}$ adatom $i$ on an $\mathrm{Ag}(111)$ island with area $S_{\text {mig }}\left[\mathrm{nm}^{2}\right]$. This scene is same as in Fig. 3 .

For the unit vectors of the incident atoms $\vec{i}$ and the surface normal $\vec{n}$ the local flux is given by

$$
f_{\mathrm{loc}}=F S_{\mathrm{mig}} \vec{i} \cdot \vec{n} / t_{\mathrm{exp}}^{\mathrm{tot}}[\text { atoms } / \mathrm{s}] .
$$

As shown in $\mathrm{V} \mathrm{A}$, most transition events relating to the island are only (1) migration on the (111) island itself, (2) a transition over the step edge by the atom $i$, and (3) a transition along the island edge by $i$ and $i^{\prime}$ since the other transitions are blocked

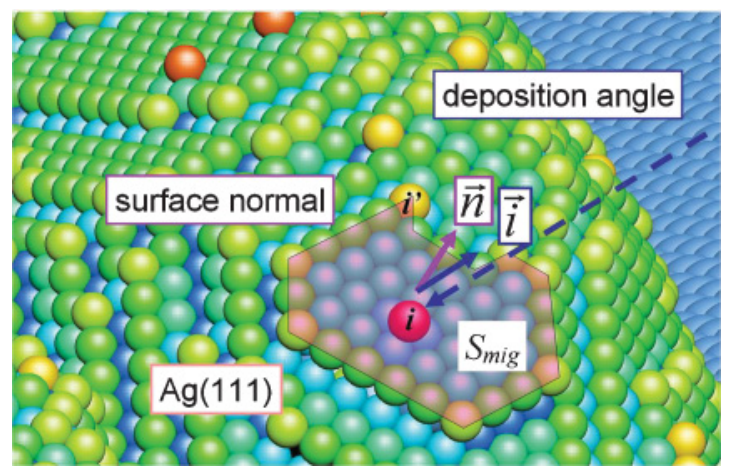

FIG. 8. (Color online) Schematic description of local flux for Ag deposition on an $\mathrm{Ag}$ (111) island. The area of the island is $S_{\mathrm{mig}} \sim$ $3.06 \mathrm{~nm}^{2} . \vec{n}$ and $\vec{i}$ are as defined in Fig. 6 .

by high potential barriers. The nondimensional time interval between two external modifications (Ag deposition) in this region is

$$
\Delta t=f_{\mathrm{loc}}^{-1} \omega_{D}^{\mathrm{Ag}} .
$$

The consistent numerator of the convergence factor $\iota$ for this situation can be estimated from (6), (29), (30), (31) and the local parameters as

$$
\frac{5.32 \times 10^{-6}}{S_{\mathrm{mig}} \vec{i} \cdot \vec{n}}<\frac{\iota}{\omega_{D}^{\mathrm{Ag}}}<\frac{5.48 \times 10^{-6}}{S_{\mathrm{mig}} \vec{i} \cdot \vec{n}}[\mathrm{~s}] .
$$

The simulation would therefore predict a convergence time for local metastable states of the order of microseconds for the situation shown in Fig. 8. In other words, the deposited atom $i$ that might arrive at a neighbor position of the atom $i^{\prime}$ would reach a stable local configuration at the latest $1.80 \mu \mathrm{s}$ for $S_{\text {mig }} \sim 3.06 \mathrm{~nm}^{2}$ after the deposition with a high probability when the surface is facing the evaporation source $(\vec{i} \cdot \vec{n}=1)$.

For the region that is not exposed to the largest flux, the surface is more bare due to more re-evaporation occurring before the next deposition event.

\section{CONCLUSION}

An atomistic, KMC simulation method has been proposed and successfully applied to metal cluster growth processes on surfaces with prepatterned ripple structures. The method consists of two essential ideas. One is the discretization of the entire process into local transition events, and the other is the classification of the transition levels with respect to the thermal fluctuation of the system. By allowing free transitions with small barriers to be considered as fluctuations, the entire system evolves as a Markov chain of equivalence class objects. The evolution dynamics of the system is promoted by the metastable level transitions. Also, contrary to some previous accelerated dynamics methods [25-27], where there are many small migration barriers, an acceleration of the system evolution can still be achieved, when the system does not require too many transition events to reach the quasiequilibrium state. In the metallic PVD example, the model predicts a convergence time of metastable states, after each metal deposition onto the surface, of $<\mu$ s order. The model 
can also predict the metal cluster growth patterns accurately with detailed atomistic transition event statistics.

An application limit of the transition level classification method may exist, due to the inability of the small transition effect to model the physics at high temperatures, but so long as the system does not require too many transition events to reach the usual fluctuation, the model provides a relatively fast (3-9 CPU days on a modern single-processor machine) estimation of the metal cluster growth process of 6-12 ML accumulation. Since the boost factor is 2290 , a traditional KMC simulation, i.e., without a boost, might require 18-54 CPU years to obtain a similar surface coverage.

These simulations can be developed further by utilizing more data from experimental measurements together with more accurate barrier calculations such as those provided by density functional theory or improved interatomic potentials. The method can also be extended to more complex systems such as multicomponent materials and grain boundaries, provided a good description of the relevant transition barriers is available.

Other modifications could include, for example, use of the nudged elastic band method [28] to calculate accurate energy barriers together with a systematic storage of data [29] so that local configurations can be easily recognized if they have previously occurred, thus accelerating the system evolution. In addition, the aesthetic algebraical structure of the Markov chain [30] itself is also a subject of interest to study, especially as an extended class 4 object of cellular automaton [21].

\section{ACKNOWLEDGMENTS}

The authors thank Mukesh Ranjan for frequent communication and advice from the experimental side, Karl-Heinz Heinig and Sibylle Gemming for helpful discussions and kindly comments on the manuscript, and Deutsche Forschungsgemeinschaft P-HE2137/4-1 for financial support.
[1] G. Braun, S. J. Lee, M. Dante, T.-Q. Nguyen, M. Moskovits, and N. Reich, J. Am. Chem. Soc. 129, 6378 (2007).

[2] N. Liu, H. Guo, L. Fu, S. Keiser, H. Schweizer, and H. Geissen, Nat. Mater. 7, 31 (2008).

[3] M. Klein and W. Shinoda, Science 321, 798 (2008).

[4] J. A. Sprague, F. Montalenti, B. P. Uberuaga, J. D. Kress, and A. F. Voter, Phys. Rev. B 66, 205415 (2002).

[5] G. Henkelman and H. Jónsson, J. Chem. Phys. 115, 9657 (2001).

[6] L. Vernon, S. D. Kenny, R. Smith, and E. Sanville, Phys. Rev. B 83, 075412 (2011).

[7] L. Vernon, S. D. Kenny, and R. Smith, Nucl. Instrum. Methods Phys. Res. B 268, 2942 (2010).

[8] M. E. J. Newman and G. T. Barkema, Monte Carlo Methods in Statistical Physics (Oxford University Press, New York, 1999).

[9] M. Strobel, K. H. Heinig, and W. Möller, Phys. Rev. B 64, 245422 (2001).

[10] A. Keller, S. Rossbach, S. Facsko, and W. Möller, Nanotechnology 19, 135303 (2008).

[11] C. M. Fortuin and P. W. Kasteleyn, Physica 57, 536 (1972).

[12] R. B. Potts, Proc. Camb. Philos. Soc. 48, 106 (1952).

[13] M. Ranjan, T. W. H. Oates, S. Facsko, and W. Möller, Opt. Lett. 35, 2576 (2010).

[14] S. Numazawa and R. Smith (unpublished).

[15] S. Numazawa, M. Ranjan, K.-H. Heinig, S. Facsko, and R. Smith, J. Phys. Condens. Matter 23, 222203 (2011).
[16] V. Rosato, M. Guillope, and B. Legrand, Philos. Mag. A 59, 321 (1988).

[17] M. Guillope and B. Legrand, Surf. Sci. 215, 577 (1989).

[18] S. V. Eremeev, A. G. Lipnitskii, A. I. Potekaev, and E. V. Chulkov, Russ. Phys. J 40, 584 (1997).

[19] R. Ferrando, Phys. Rev. Lett. 76, 4195 (1996).

[20] Y. F. Zhukovskii, E. A. Kotomin, P. W. M. Jacobs, and A. M. Stoneham, Phys. Rev. Lett. 84, 1256 (2000).

[21] S. Wolfram, A New Kind of Science (Wolfram Media, Champaign, IL, 2002).

[22] R. M. Ferullo, G. R. Garda, P. G. Belelli, M. M. Branda, and N. J. Castellani, J. Mol. Struct.: Theochem 769, 217 (2006).

[23] M. Ranjan (private communication).

[24] J. Petersen and S. G. Mayr, J. Appl. Phys. 103, 23520 (2008).

[25] M. R. Sørensen and A. F. Voter, J. Chem. Phys. 112, 9599 (2000).

[26] F. Montalenti, A. F. Voter, and R. Ferrando, Phys. Rev. B 66, 205404 (2002).

[27] A. Chatterjee and A. F. Voter, J. Chem. Phys. 132, 194101 (2011).

[28] N. Castin and L. Malerba, J. Chem. Phys. 132, 074507 (2010).

[29] F. G. Djurabekova, R. Domingos, G. Cerchiara, N. Castin, E. Vincent, and L. Malerva, Nucl. Instrum. Methods Phys. Res. B 255, 8 (2007)

[30] P. Bidigare, P. Hanlon, and D. Rochmore, Duke Math. J. 99, 135 (1999). 